\title{
Cost-effectiveness analysis of the introduction of the Pneumococcal Conjugate Vaccine (PCV-13) in the Egyptian national immunization program, 2013
}

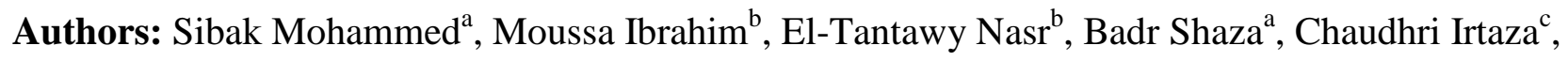 \\ Allam Essam $^{\mathrm{d}}$, Baxter Louise ${ }^{\mathrm{e}}$, Abo Freikha Saiyed ${ }^{\mathrm{f}}$, Hoestlandt Céline ${ }^{\mathrm{g}}$, Lara Carlos ${ }^{\mathrm{g}}$, Hajjeh \\ Rana $^{\mathrm{h}}$, Munier Aline ${ }^{\mathrm{g}}$.
}

\author{
Affiliations: \\ ${ }^{\text {a }}$ Expanded Program on Immunization, Ministry of Health, Egypt \\ ${ }^{\mathrm{b}}$ World Health Organization, Country Office, Egypt \\ ${ }^{\mathrm{c}}$ World Health Organization, Regional Office for the Eastern Mediterranean, Cairo, Egypt \\ ${ }^{\mathrm{d}}$ UNICEF, Egypt \\ ${ }^{\mathrm{e}}$ Department of Health Services Research and Policy, London School of Hygiene and Tropical \\ Medicine, London, UK \\ ${ }^{\mathrm{f}}$ Gharbiya Health Directorate, Egypt \\ g Agence de Médecine Préventive, Paris, France \\ ${ }^{\mathrm{h}}$ Centers for Disease Control and Prevention (CDC), Atlanta, USA
}

*Corresponding author: Dr Mohammed Sibak, email:sibakmohammed@gmail.com, telephone number: +201222655041 


\section{Introduction}

Pneumonia remains the leading cause of deaths in children aged 1-59 months worldwide, representing $14.1 \%$ of deaths, and Streptococcus pneumoniae is the most common cause of vaccine-preventable severe pneumonia and death $[1,2]$. Two new pneumococcal conjugate vaccines (PCVs) are currently available and are being introduced in several countries. They target either 10 (PCV-10) or 13 (PCV-13) of the most prevalent serotypes. Several clinical trials have proven their safety and efficacy in preventing invasive pneumococcal disease (IPD), pneumonia, and acute otitis media (AOM) [3-13], and they are currently used in more than 100 countries around the world, including many low- and middle-income countries. In addition, they can be easily integrated in the Expanded Program on Immunization (EPI) schedule, and were shown to be safely co-administered with other vaccines without interference. In its 2012 position paper, the World Health Organization (WHO) recommended the inclusion of PCVs in childhood immunization programs worldwide [14].

Pneumonia is considered one of the most important causes of morbidity and mortality in children under 5 years in Egypt; the incidence of pneumococcal pneumonia estimated at 529 per 100,000 in 2010, according to a recent systematic review [15]. In Egypt, local data on the incidence of pneumonia and prevalence of the various serotypes are limited. However, a national, laboratorybased sentinel-surveillance study of bacterial meningitis conducted between 1998 and 2004 showed that approximately $36 \%$ of isolates from patients with bacterial meningitis were due to $S$.

pneumoniae among children under 5 [16]. A study looking at serotype distribution of these isolates revealed that only about a third of the serotypes were included in the PCV7 vaccine, but no data are available on distribution of serotypes that cause severe pneumonia or invasive pneumococcal disease other than meningitis [17]. 
In Egypt, the National Immunization Technical Advisory Group (NITAG) is the entity

responsible for immunization policy and the decision-making process. The Haemophilus influenzae type B (Hib) vaccine was introduced in early 2014 and is combined with DTP and Hepatitis B in the form of a pentavalent vaccine. The Ministry of Health and Population (MOHP) is considering introducing PCV in its immunization program. The country chose to study PCV13 because this vaccine provides better coverage of pneumococcal strains circulating in Egypt as compared to PCV10. Consequently, the EPI conducted a cost-effectiveness analysis (CEA) in collaboration with the ProVac International Working Group (IWG) through the Agence de Médecine Préventive (AMP), and the WHO regional office for the Eastern Mediterranean. The aim was to help the EPI staff build cost-effectiveness analysis skills and provide decision-makers with needed evidence for introducing this vaccine into Egypt's routine vaccination schedule.

Objectives of this study were to assess health outcomes, incremental vaccination program costs, averted healthcare system costs, the cost-effectiveness of introducing PCV13 in the national immunization program, and to compare these measures against no vaccination.

\section{Methods}

\subsection{General description of the model}

The analysis was performed using the TRIVAC model (version 2.0) developed at the London School of Hygiene and Tropical Medicine (LSHTM) with support from the Pan American Health Organization's (PAHO's) ProVac Initiative and the Global Alliance for Vaccines and Immunization (GAVI) [18]. The TRIVAC model is a static cohort model, developed in EXCEL and designed for use in low- and middle-income countries. The model structure has been described in detail elsewhere [19]. Briefly, four diseases were included in the model: all-cause AOM, pneumococcal (Spn) pneumonia cases, Spn meningitis and Spn non- 
pneumonia non-meningitis (NPNM); these are other forms of invasive disease, primarily sepsis and bacteremia. The model uses data such as demographics, burden of disease, program coverage and efficacy of PCV13, serotype coverage, waning protection, health resource utilization, and costs of both pneumococcal disease vaccination and treatment of children under 5 .

Whenever possible and available, local data from Egypt was used. If local data was not available, regional or global data was reviewed by a panel of national and international experts and used for the study. Experts' opinions were obtained during professional EPI meetings in high committees, including members of the MOHP, professors of pediatrics, the head of the polio eradication certification committee, and senior medical epidemiologists. All of them had to reach a consensus.

The model provides outcomes about the number of pneumococcal disease cases averted, outpatient visits and admissions averted, deaths averted, the costs incurred to include the vaccine in the national schedule, and health services costs prevented due to cases of disease averted. The model also provides information on life-years saved, disability-adjusted life-years (DALYs) averted throughout the lifetime of that cohort, and an incremental cost- effectiveness ratio (ICER), with the cost expressed in U.S. dollars per DALY averted [19]. To estimate DALYs averted, the model considered the WHO Global Burden of Disease (GBD) project disability weights for each disease (WHO GBD 1990).

The cost-effectiveness of PCV13 as opposed to the no-vaccination scenario was determined as follows: $\mathrm{ICER}=($ cost of the vaccination program - health care costs saved through disease prevention) / DALYs averted. [20]

Using the WHO recommended threshold [21], if the ICER of the vaccine is less than the gross domestic product (GDP) per capita, it is considered highly cost-effective; if it is between 1x and 3x GDP per capita, it is considered cost-effective; and if it is more than 3 GDP per capita, it is not cost-effective. 
Multivariate sensitivity analysis was used to determine how changes in values of one or

74

75

76

77

78

79

80

81

82

83

84

85

86

87

88

89

90

91

92

93

94 several inputs simultaneously would affect the outcome under a given set of assumptions. Key

drivers of the model results were tested, as were parameters that had uncertain values or came from multiple sources.

Two alternatives were compared: (1) introduction of PCV13 into the current national immunization program and (2) no introduction of PCV13. The total outcomes over 10 cohorts (2014-2023) from birth to 5 years were analyzed. The discount rate was defined at $3 \%$, as recommended by WHO [20]. Age weighting was not considered in the analysis. The study was performed from the government perspective; public health providers were included (clinics, primary, secondary and tertiary hospitals).

\subsection{Demographic data}

The in-country population in mid-2013 was estimated at 86 million, with the total population of children younger than 5 at 9 million (WHO, 2010). Demographic data used to populate the model was derived from the 2006 census done by the Central Authority for Public Mobilization and Statistics (CAPMAS). In 2012, live births were estimated at 2.6 million. The mortality rates for infants and children under 5 were 6.5 per 1,000 and 20.4 per 1,000, respectively. Life expectancy was estimated at 73 years in 2012 (United Nations Population Division [UNPOP], 2010 revision).

\subsection{Disease burden}

Data on incidence and deaths for each clinical syndrome was obtained from various sources (Table 1). For all-cause AOM, data was based on the incidence of acute respiratory infections reported in Rudan 2004 [22], and then was doubled to assume an AOM incidence of 29,300 per 100,000. Another study conducted in Boston [23], which showed an incidence of 90,000 per 100,000 cases of clinical otitis in children younger than 5, was used for the high- 
incidence scenario. It was assumed that AOM does not require hospitalization and that it is not associated with fatal cases.

For pneumococcal pneumonia, a recent publication from Rudan (2013) [15] was used, based on an epidemiological model that takes into account each country's profile and incorporates data from national Demographic and Health Surveys (DHS) for risk factors of pneumonia. It gave an estimate of 47,625 new cases of pneumococcal pneumonia in Egypt, which translates to 529 per 100,000 children under age 5. Data from the GBD 2000 [24] was used in the high-incidence scenario, with an incidence of Spn pneumonia of 980 per 100,000. On the national level, deaths due to pneumococcal pneumonia reported by the Maternal and Child Health department of the MOHP in 2012 were estimated as 1,200, which corresponds to 40\% of all-cause pneumonia deaths in Egypt. The case-fatality ratio (CFR) - i.e., the number of deaths divided by the number of new cases of this disease - was estimated at 2.5\%. In Rudan 2013 [15], 1,570 pneumonia deaths due to Spn were reported in Egypt, corresponding to a CFR of 3\%; this was used in the high-CFR scenario. The annual incidence of pneumococcal meningitis, based on the GBD project [24] estimates for the Middle East, was 14 per 100,000. Local data was also available from the Epidemiology Surveillance Unit, MOHP, which registered 35 cases (children younger than 5) in 2012, an incidence of 0.4 per 100,000. (This probably underestimates the real disease burden, so it was tested in a low-burden scenario.) The CFR for Spn meningitis was estimated at 7.9\%, based on the Eastern Mediterranean regional study from Teleb 2013 [25]. A 15\% rate was used in the high-burden scenario, based on a local surveillance study of bacterial meningitis in Egypt [26]. No local data was available on NPNM disease, so the 2000 GBD study was used instead; the incidence was 84 per 100,000 with a CFR of $4.6 \%$.

Local data on sequelae in meningitis survivors is scarce, so data from a recent global systematic review [27] was used, showing a risk of $20.2 \%$ for major single sequelae among survivors and a risk of $4.5 \%$ for major multiple sequelae following pneumococcal meningitis. 
121 Disability weights of 0.28 for pneumonia and 0.62 for meningitis were applied [28]. For NPNM

122 diseases, pneumonia disability weights were used as a proxy. Weighted disability averages were

123 calculated for sequelae based on the reported global distribution of sequelae syndromes [27] and

124 standard disability weights for conditions similar to each syndrome [28]. We used 0.24 for single

125 pneumococcal sequelae and 0.63 for multiple pneumococcal sequelae [29]. The mean duration of

126 illness was 6 days for AOM and NPNM and 10 days for Spn pneumonia and meningitis, based on

127 local expert opinion.

128

129

130

131

132

133

134

135

136

137

138

139

140

141

142

143

\subsection{Vaccine coverage and efficacy}

The vaccine (PCV13) is presented as a 1-dose vial, Prevnar13® (Pfizer, NY). A 3-dose schedule without booster was considered, administered at 2, 4, and 6 months, together with the pentavalent vaccine 3 doses, as per the current EPI schedule. In the year of introduction, we assumed coverage rates for doses 1, 2, and 3 at 96.7\%, 96.6\%, and 96.5\%, respectively, based on rates achieved in 2012 for the DTP3 (EPI, MOHP reports). The model also takes into account the timeliness of vaccination [30] (Table 2).

For all-cause AOM, full-dose efficacy data was derived from a meta-analysis by Pavia et al. in 2009 [31] that used efficacy rates from several trials conducted on PCV; the analysis demonstrated an efficacy of 6\% against all-cause AOM. A meta-analysis in the Cochrane Review in 2009 [32] showed $81 \%$ vaccine efficacy against invasive pneumococcal disease, which was used for Spn pneumonia, meningitis, and NPNM. The reduced efficacy was estimated based on the ratio between one and two doses, or two and three doses, as reported by Mahon in 2006 [33] and multiplied by the full-dose efficacy. For AOM, one- and two-dose efficacy were estimated at $3 \%$ and 5.5\%, respectively; for pneumococcal pneumonia, meningitis, and NPNM, one- and two-dose efficacy were estimated at $41 \%$ and $74.5 \%$, respectively (Table 3 ). 


\subsection{Serotype coverage}

\subsection{Indirect effects of vaccination}

In the base-case scenario, we assumed a conservative $110 \%$ 'herd effect' multiplier. The multiplier accounts for herd effects of the vaccine in the under-5 age group. Because evidence on the herd effect of PCV13 is lacking, the $110 \%$ multiplier is an assumption, and due to the uncertainty surrounding this input, the team opted for a conservative assumption in the base case. In another scenario, the team used a "high" herd effect multiplier of $125 \%$, based on evidence from the USA in PCV7 in order to account for indirect effects; In this latter study, the total impact of the vaccine - $94 \%$ - was divided by the direct impact (i.e. the efficacy observed in studies in the USA, 75\%) in order to derive a $125 \%$ herd effect multiplier for the model [35]. A serotype replacement of $5.5 \%$ per year was included, based on a study of PCV7 conducted in England and Wales. [36]. Finally, we assumed a 5\% annual decrease in vaccine efficacy (waning efficacy). In the model, the 'relative coverage of death' parameter is used to adjust the vaccination program coverage to account for high risk children who do not receive the vaccine. As a proxy for this value, we have used the vaccine coverage from the lowest wealth quintile, divided by the total coverage, based on what is reported in the Egyptian DHS (99\%).

Other estimates of these impact assumptions, which are key drivers of the model, were tested in the scenario analysis.

\subsection{Vaccination program costs}


167 the vaccine were adopted, priced it at US\$ 14.24 , plus an additional $3 \%$ for handling, $1 \%$ for

168 delivery, and $1 \%$ for wastage (Table 4). Other costs include safety boxes (US\$ 0.60 ) and the

169 administering syringe for each dose (US\$ 0.06). It was assumed that the price would decrease $3 \%$

170 per year.

Based on the opinion of EPI experts, US\$ 1 per dose-about $7 \%$ of the vaccine per-dose

price—was assumed for incremental system costs, including cold chain, transportation, training,

173 and social mobilization such as awareness campaigns. (Based on 2013 exchange rates, US\$ 1 is

174 equivalent to 7 Egyptian pounds [EGPs]. US\$ 14 is approximately EGP 100.)

175 Other scenarios with lower and higher prices were also tested, as vaccine price is a key

176 driver of the result.

\subsection{Health resource utilization}

178

General access to care in Egypt is about 70\%, according to the household health expenditure and utilization survey 2009/2010 [37]. But because it was assumed that not every child with access to care would visit a healthcare provider, we set outpatient visitation rates for all-cause AOM at 50\%. For Spn pneumonia and meningitis, we assumed an outpatient visitation rate of $80 \%$; for $S p n$ NPNM, we assumed an outpatient visitation rate of 50\%. In order to estimate the number of hospitalizations due to pneumococcal pneumonia, the number of new severe cases of Spn pneumonia from Rudan 2013[15] was used (i.e., 14,318), multiplied by the proportion of hospitalizations of severe cases $(80 \%)$. This was then divided by the number of new cases $(47,625)$ to obtain a $24 \%$ utilization rate. Based on expert opinion, it was assumed that all meningitis cases are hospitalized, since nearly all diagnosed cases are admitted; it was also assumed that $75 \%$ of Spn NPNM cases are admitted to hospitals. 
expert opinion. They are reported in Table 5.

191

192

193

194

195

196

197

198

199

200

201

202

203

204

205

206

207

208

209

210

211

212

213

\subsection{Health services costs}

All costs analyzed in this study are the cost of the four illnesses in the public sector whose treatment is funded by the government.

Resource utilization was estimated by surveying 100 physicians from 20 hospitals located

in 4 governorates (Cairo, Giza, Bani-Sweif and Gharbiya) representing three geographical areas of Egypt (traditionally upper Egypt, lower Egypt and greater Cairo) with socioeconomic variations. Physicians were specialists in different areas (general pediatricians, neurologists, oto-rhinolaryngologists, and physicians in fever and respiratory diseases). The physicians were asked about the usual treatment in daily practice for a given syndrome. The weighted unit cost (costs for medicines, laboratory tests, and x-rays) for each intervention or practice was registered.

The average costs of outpatient and inpatient treatments were estimated from these field surveys when possible, based on the proportion of complicated and uncomplicated cases for each disease. An average of the government costs obtained was then computed by disease and by health provider (Table 5).

Because NPNM is not definitely diagnosed, it is treated like pyrexia of unknown origin, and corresponding costs were used.

For outpatient all-cause AOM and Spn NPNM, no local information could be obtained from the survey; instead we used the default data from WHO-CHOICE. (WHO-CHOICE is an initiative that provides policy makers with the evidence for deciding on the interventions and programs which maximize health for the available resources. (Available at: http://www.who.int/choice/en/.)

For admissions, a bed-cost of US\$ 100 for the total length of stay was assumed, based on national pediatric experts. That sum includes cost of residence, service, and logistics. Reference 
214 prices for drugs used in hospitalization were obtained from information provided by surveyed 215 hospitals. The price used is the hospitals' purchasing price.

The annual cost of major single and multiple sequelae from meningitis was not included in

217 the base-case scenario because no significant data from Egyptian sources was available. However,

218 to test the impact of this parameter, some annual household costs corresponding to Egypt's GDP

219 per capita (based on Griffiths et al., 2012 [38]) were included in a tested scenario.

\section{3. Results}

\subsection{Impact on pneumococcal disease burden and health service utilization}

224 hospitalizations (Tables 6 and 7).

The introduction of PCV13 vaccine would be cost-effective with an incremental costthe WHO recommended threshold [21] (Table 7). This corresponds to 1.2 x GDP per capita in (10 years) is US\$1,089,181,321 (approximately US\$1.1 billion).

\subsection{Sensitivity analysis}

In order to test the robustness of the base-case results, we ran 18 different scenarios in the model and adjusted various parameters that were either key drivers of the model or for which we

234 had uncertain values or different values depending on the source. Each of the 18 scenarios resulted 
in an ICER that was still cost-effective (highly cost-effective for three of the scenarios) from the government perspective (Figure 1). As part of this analysis, two 'extreme' scenarios were tested:

- The most favorable scenario comprised a low vaccine price and a high incidence and casefatality ratio: US\$1,404 per DALY averted (highly cost-effective).

- The least favorable scenario comprised a high vaccine price and a low incidence and casefatality ratio: US\$ 7,833 per DALY averted (cost-effective).

Results from the government perspective should be considered robust because, despite testing a significant uncertainty, it was demonstrated that at a minimum the PCV13 intervention would be cost-effective.

\section{Discussion}

Primary results show that introduction of PCV13 would be a cost-effective intervention in Egypt.

In low- and middle-income countries, evidence-based decision-making and economic studies in particular are still limited, often due to a lack of relevant data or a systematic process. The ProVac IWG was an opportunity for some of these countries to be exposed to the methodology and gain support from partners to carry out their own analysis and promote evidence-based decision-making, especially regarding new vaccine introductions. This is especially relevant in the context of limited resources and in light of the high cost of the new vaccines (as compared to traditional vaccines of the EPI), particularly for middle-income countries that do not benefit from GAVI support [39].

Many of the published cost-effectiveness studies in these settings come from Latin America and the Caribbean, where the ProVac Initiative was first launched $[18,40]$. They have shown that, compared to no vaccination, either PCV10 or PCV13 would be cost-effective 
[42]; Colombia, US\$1,837 per DALY for PCV10 [43]). In African low-income countries, PCV13 vs. no vaccination was cost-effective in Gambia, with an ICER of US\$ 570 per DALY corresponding to $1.6 \mathrm{x}$ GDP per capita [44]; and highly cost-effective in Kenya, with an ICER of US\$ 47 per DALY [45].

Our analysis has a few limitations. First, estimating some costs that are not directly associated with the healthcare system is difficult; opportunity costs such as parents' loss of productivity when taking care of sick children could not be included. Consequently, even though we were able to obtain partial information on household costs, the societal impact was not described. Second, data from the private sector was not available and private health facilities were not considered, even though some patients may go to private clinics. Third, sequelae costs incurred by the government were not included in the base-case analysis because no Egyptian data was available. This would affect the overall ICER by underestimating cost savings to the health system. Lastly, for a more comprehensive analysis, results would need to be strengthened by collecting additional accurate prospective data, and if possible, by adding the societal point of view.

Despite these limitations, the government perspective was the perspective of most interest because in Egypt, $70 \%$ of the population receives healthcare services from governmental institutions. The study allowed us to document the direct medical costs borne by the government (the public health sector) if the vaccine were introduced nationwide. Also, whenever possible, national or regional data were used. Incomplete data was complemented by international literature and meta-analyses, and all data used to populate the model was validated by a multidisciplinary team of national and international experts, including different institutions and specialists in various fields, including epidemiologists, health economists, public health and immunization experts. Our results were shown to be robust. The scenario analysis allowed us to test several scenarios, and all of them showed that introduction of PCV13 would at least be cost-effective. 
Three scenarios showed PCV13 to be highly cost-effective. The scenario with the highest ICER was the least favorable one for the vaccine introduction. Therefore, in the base case, introduction of PCV13 in Egypt is cost-effective from the government point of view.

Finally, our results are likely to be conservative: we did not include societal and sequelae costs and the chosen herd effect multiplier of $110 \%$ is lower than what might be expected because it only took into account children under 5. Had older age groups been considered, it almost surely would lead to a more cost-effective analysis from the societal perspective.

\section{Conclusion}

Introduction of PCV13 would represent a high-impact public health intervention for Egypt and would respond to the NITAG resolution on reducing pneumonia cases and pneumococcal deaths. Strengthening epidemiological and laboratory surveillance will be of fundamental importance when making a decision about introducing this vaccine into the schedule, to measure the impact of the intervention. Intersectoral and multidisciplinary work within the framework of the MOHP allowed us to revise available data, producing solid evidence and facilitating evidence-based decision-making. Cost-effectiveness analysis could be an important part of a strong evidence-based decision-making process and needs to be considered for various public health interventions.

\section{Acknowledgements}

The authors are grateful to: Andrew Clark and Colin Sanderson (LSHTM), who provided support using and interpreting the TRIVAC model; Julia Blau, who coordinated the implementation of the project at AMP; our colleagues Antoine Durupt, Chloé Thion, and Aude Bruyas at AMP; Gabriela Felix (PAHO), who coordinated the ProVac IWG at PAHO; Diane 
306 Coraggio, who provided support to country teams regarding the effective communication of

307 evidence to stakeholders; Dr Nadia Teleb, Regional Advisor, VPI for the Eastern Mediterranean

308 (WHO-EMRO); Miloud Kaddar (formerly WHO-HQ); Dr. Loreis Ramses, Cairo governorate

309 surveillance office; Dr. Mona Toson, Bani-Sweif governorate surveillance office, and Mrs. Heba

310 Wali, Vacsera, holding company.

311

312 Conflicts of interest

313 All authors declare no conflict of interest.

$315 \quad$ Funding

This study is part of the ProVac IWG and was funded by a grant from the Bill and Melinda

317 Gates Foundation to the PAHO. The Agence de Médecine Préventive (AMP) was PAHO's sub-

318 grantee and implemented the project in the WHO Eastern Mediterranean region (EMRO) and the

319 European region (EURO).

320

321 
1. Liu L, Johnson HL, Cousens S, Perin J, Scott S, Lawn JE, et al., for the Child Health Epidemiology Reference Group of WHO and UNICEF. Global, regional, and national causes of child mortality: an updated systematic analysis for 2010 with time trends since 2000 . The Lancet 2012; 379:2151-61.

2. Walker CL, Rudan I, Liu L, Nair H, Theodoratou E, Bhutta ZA, et al. Global burden of childhood pneumonia and diarrhea. The Lancet 2013; 381:1405-16.

3. Black S, Shinefield H, Fireman B, Lewis E, Ray P, Hansen J, et al. Efficacy, Safety and immunogenicity of heptavalent pneumococcal conjugate vaccine in children. The Ped Infect Dis J., 2000; 19(3):187-95.

4. Prymula R, Peeters P, Chrobok V, Kriz P, Novakova E, Khol I, et al. Pneumococcal capsular polysaccharides conjugated to protein $\mathrm{D}$ for prevention of acute otitis media caused by both Streptococcus pneumoniae and non-typable Haemophilus influenzae: a randomized doubleblind efficacy study. The Lancet 2006; 367:740-748.

5. Vesikari T, Wysocki J, Chevallier B, Karvonen A, Czajka H, Arsène JP, et al. Immunogenicity of the 10-Valent Pneumococcal non-typeable Haemophilus influenzae Protein D Conjugate Vaccine (PHiD-CV) Compared to the Licensed 7vCRM Vaccine. The Ped Infect Dis J. 2009; 28 (4): S66.

6. Bermal N, Szenborn L, Chrobot A, Alberto E, Lommel P, Gatchalian S, et al. The 10-Valent Pneumococcal non-typeable Haemophilus influenzae Protein D Conjugate Vaccine (PHiDCV) coadministered with DTPw-HBV/Hib and Poliovirus vaccines: Assesment of Immunogenicity. The Ped Infect Dis J. 2009; 28 (4): S89.

7. Chevalier B, Vesikari T, Brzostek J, Knuf M, Bermal N, Aristegui J, et al. Safety and Reactogenicity of the 10-Valent Pneumococcal non-typeable Haemophilus influenzae Protein 
D Conjugate Vaccine (PHiD-CV) when coadministered with Routine Childhood Vaccines. The Ped Infect Dis J. 2009; 28 (4): S109.

8. Omeñaca F, Constantopoulos A, Merino JM, Tejedor JC, Athanasiadou F, Francois N, et al. Vaccination of preterm infants with the 10-Valent Pneumococcal non-typeable Haemophilus influenzae Protein D Conjugate Vaccine (PHiD-CV). The Ped Infect Dis J. 2009; 28 (6): e190.

9. Esposito S, Tansey S, Thompson A, Ferrera G, Maida A, Bona G, et al. Safety and immunologic non-inferiority of 13-Valent Pneumococcal Conjugate Vaccine given as a 3dose series with routine vaccines in healthy children. The Ped Infect Dis J. 2009; 28 (6): e169.

10. Diez-Domingo J, Gurtman A, Bernaola E, Gimenez-Sanchez F, Martinon-Torres F, PinedaSolas V, et al. Safety and Immunogenicity of 13-Valent Pneumococcal Conjugate Vaccine in healthy infants and toddlers receiving routine vaccination in Spain. The Ped Infect Dis J. 2009; 28 (6): e167.

11. Grimpel E, Laudat F, Baker SA, Sekaran C, Gruber WC, Scott DA. Safety and Immunogenicity of a 13-Valent Pneumococcal Conjugate Vaccine given with routine pediatric vaccination to healthy children in France. The Ped Infect Dis J. 2009; 28 (6): e174.

12. Hughes JY, Snape MD, Klinger CL, Daniels E, Layton H, John TM, et al. Immunogenicity of booster doses of 13-Valent Pneumococcal Conjugate and HIB/MENC Vaccines given at 12 months of age in the UK. The Ped Infect Dis J. 2009; 28 (6):e177.

13. Martinon-Torres F, Gimenez-Sanchez F, Gurtman A, Bernaola E, Diez-Domingo J, Carmona A, et al. Safety and Immunogenicity of 13-Valent Pneumococcal Conjugate Vaccine given with Meningococcal C Conjugate and other pediatric vaccinations in Spain. The Ped Infect Dis J. 2009; 28 (6): e185.

14. World Health Organization. Pneumococcal vaccines WHO position paper. WER 2012; 87: 129- 
15. Rudan I, O’Brien K.L, Nair H, Liu L, Theodoratou E, Qazi S, et al. Epidemiology and etiology of childhood pneumonia in 2010: estimates of incidence, severe morbidity, mortality, underlying risk factors and causative pathogens for 192 countries. J Glob Health. 2013. 3(1):

16. Afifi S, Wasfy MO, Azab MA, Youssef FG, Pimentel G, Graham TW, et al. Laboratory-based surveillance of patients with bacterial meningitis in Egypt (1998 - 2004), Eur J Clin Microbiol Infect Dis. 2007; 26: 331-340.

17. Wasfy MO, Pimentel G, Abdel-Maksoud M, Russell KL, Barrozo CP, Klena JD, et al.

18. Jauregui B, Sinha A, Clark AD, Bolanos BM, Resch S, Toscano CM, et al. Strengthening the

20. WHO IVB. Guide for standardization of economic evaluations of immunization programmes.

\section{Clark A, Jauregui B, Griffiths U, Janusz C, Bolaños-Sierra B, Hajjeh R, et al. TRIVAC} decision-support Model for evaluating the cost-effectiveness of Haemophilus influenzae type b, pneumococcal and rotavirus vaccination. Vaccine 2013, Suppl 3: C19-29. 2008. WHO/IVB/08.14.

21. Sachs J. Macroeconomics and health: investing in health for economic development. World Health Organization; Geneva: 2001.

22. Rudan I, Tomaskovic L, Boschi-Pinto C, Campbell H. WHO CHERG. Global estimate of the incidence of clinical pneumonia among children under five years of age. Bulletin of the World Health Organization. 2004; 82 (12): 895-903.

23. Teele DW, Klein JO, Rosner B, and the Greater Boston Otitis Media Study Group. 
Epidemiology of Otitis Media During the First Seven Years of Life in Children in Greater Boston: A Prospective, Cohort Study. J Infect Dis.1989; 160(1): 83-93.

398

399

400

401

402

403

404

405

406

407

408

409

410

411

412

413

414

415

416

417

418

419

420
24. O'Brien KL, Wolfson LJ, Watt JP, Henkle E, Deloria-Knoll M, McCall N, et al. Burden of disease caused by Streptococcus pneumoniae in children younger than 5 years: global estimates. The Lancet. 2009; 374:893-902.

25. Teleb N, Pilishvili T, Beneden C.V, Ghoneim A, Amjad K, Mostafa A, et al. Bacterial Meningitis surveillance in the Eastern Mediterranean Region, 2005-2010: Successes and Challenges of a Regional Network, The Journal of Pediatrics 2013, Vol. 163, Supplement 1, S25-S31.

26. Youssef FG, El-Sakka H, Azab A, Eloun S, Chapman GD, Ismail T, et al. Etiology, Antimicrobial Susceptibility Profiles, and Mortality Associated with bacterial meningitis among children in Egypt, Annals of Epidemiology 2004, 14(1): 44 - 48.

27. Edmond K, Clark A, Korczak V, Sanderson C, Griffiths U, Rudan I. Global and regional risk of disabling sequelae from bacterial meningitis: a systematic review and meta-analysis. Lancet Inf Dis. 2010; 10: 317-28.

28. Murray CJL LA. The global burden of disease: a comprehensive assessment of mortality and disability from diseases, injuries and risk factors in 1990 and projected to 2020. Cambridge: Harvard University Press. 1996.

29. Griffiths U. Haemophilus Influenzae type b vaccine in low- and middle-income countries: impact, costs and incremental cost-utility ( $\mathrm{PhD}$ submitted to University of London). 2012

30. Clark A and Sanderson C. Timing of children's vaccination in 45 low-income and middleincome countries: an analysis of survey data. The Lancet. 2009; 373: 1543-49.

31. Pavia M, Bianco A, Nobile C, Marinelli P, Angelillo I. Efficacy of Pneumococcal Vaccination in Children Younger Than 24 Months: A Meta-Analysis. Pediatrics. 2009; 123 (6): 1103-10.

32. Lucero MG, Dulalia VE, Nillos LT, Williams G, Parreño RA, Nohynek H, et al. Pneumococcal 
conjugate vaccines for preventing vaccine-type invasive pneumococcal disease and X-ray defined pneumonia in children less than two years of age. Cochrane Database, 2009, Review. CD004977.

33. Mahon BE, Hsu K, Karumuri S, Kaplan SL, Mason Jr EO, Pelton SI. Effectiveness of abbreviated and delayed 7-valent PCV dosing regimens, Vaccine, 2006; 24(14): 2514-2520.

34. Johnson HL, Deloria-Knoll M, Levine OS, Stoszek SK, Freimanis Hance L, Reithinger R, et al. Systematic Evaluation of Serotypes Causing Invasive Pneumococcal Disease among Children Under Five: The Pneumococcal Global Serotype Project. PLoS Med 2010; 7(10): e1000348. doi:10.1371/journal.pmed.1000348.

35. Centers for Disease Control and Prevention. Invasive pneumococcal disease in children 5 years after conjugate vaccine introduction - Eight states, 1998 - 2005. Morbidity and Mortality Weekly Report (MMWR). February 15, 2008/ 57 (06): pp 144-148).

36. Miller E, Andrews NJ, Waight PA, Slack MP, George RC. Herd immunity and serotype replacement 4 years after seven-valent pneumococcal conjugate vaccination in England and Wales: an observational cohort study. Lancet 30-Infect Dis. 2011; 11: 760-8

37. USAID Egypt. Egypt household health expenditure and utilization survey 2009/2010 for Health Systems 20/20 Project. November 2011.

38. Griffiths U, Dieye Y, Fleming J, Hajjeh R, Edmond K. Costs of Meningitis Sequelae in Children in Dakar, Senegal. Pediatr Infect Dis J 2012; 31: e189-e195.

39. Nakamura MM, Tasslimi A, Lieu TA, Levine O, Knoll MD, Russell LB, et al. Costeffectiveness of child pneumococcal conjugate vaccination in middle-income countries. Int Health. 2011; 3:270-81.

40. Andrus JK, Walker DG. Evidence base for new vaccine introduction in Latin America and the Caribbean. Vaccine. 2013; 31 Suppl 3:C2-3. 
445 41. Urueña A, Pippo T, Betelu MS, Virgilio F, Giglio N, Gentile A et al. Cost-effectiveness

446 analysis of the 10- and 13-valent pneumococcal conjugate vaccines in Argentina. Vaccine

$447 \quad 2011 ; 29: 4963-7$.

448 42. Sartori AM, de Soárez PC, Novaes HM. Cost-effectiveness of introducing the 10-valent

449 pneumococcal conjugate vaccine into the universal immunisation of infants in Brazil. J

$450 \quad$ Epidemiol Community Health. 2012; 66:210-7.

451 43. Castañeda-Orjuela C, Alvis-Guzmán N, Velandia-González M, De la Hoz-Restrepo F. Cost-

452 effectiveness of pneumococcal conjugate vaccines of 7,10 , and 13 valences in Colombian

$453 \quad$ children. Vaccine 2012; 30:1936-43.

454 44. Kim SY, Lee G, Goldie SJ. Economic evaluation of pneumococcal conjugate vaccination in

455 The Gambia. BMC Infect Dis. 2010; 10: 260.

456 45. Ayieko P, Griffiths UK, Ndiritu M, Moisi J, Mugoya IK, Kamau T et al. Assessment of health

457 benefits and cost-effectiveness of 10-valent and 13-valent pneumococcal conjugate vaccination

458 in Kenyan children. PLoS One 2013;8(6):e67324. doi: 10.1371.

459

460 Figure 1. Cost-effectiveness of introducing PCV13 and sensitivity analysis (government

461 perspective)

462 
Table 1. Input parameters for estimating disease burden

\begin{tabular}{|c|c|c|c|c|}
\hline \multirow[t]{2}{*}{ Parameter } & \multirow[t]{2}{*}{ Estimate } & \multicolumn{2}{|c|}{ Scenarios } & \multirow[t]{2}{*}{ Source/s } \\
\hline & & Low & High & \\
\hline \multicolumn{5}{|c|}{ Annual incidence per 100,000 aged $1-59 \mathrm{~m}$ : } \\
\hline All-cause acute otitis media & 29,305 & 29,305 & 90,000 & Rudan et al. 2004 (22) \\
\hline Pneumococcal pneumonia cases & 529 & 476 & 980 & Rudan et al. 2013 (15) \\
\hline Pneumococcal meningitis & 14.0 & 0.4 & 14.3 & Epidemiology surveillance unit, MoHP \\
\hline Pneumococcal NPNM & 84.1 & 63.8 & 106.2 & GBD 2000 \\
\hline \multicolumn{5}{|c|}{$\%$ case fatality ratios (CFRs) in ages $1-59 \mathrm{~m}:^{a}$} \\
\hline Pneumococcal pneumonia cases & $2.50 \%$ & $2.23 \%$ & $3.00 \%$ & Maternal and child Health Department, MoHP 2012 \\
\hline Pneumococcal meningitis & $7.9 \%$ & $7.90 \%$ & $15.00 \%$ & Teleb et al. 2013 (25) \\
\hline Pneumococcal NPNM & $4.6 \%$ & $4.18 \%$ & $5.02 \%$ & GBD 2000 \\
\hline \multicolumn{5}{|c|}{$\%$ sequelae in pneumococcal meningitis survivors } \\
\hline$\%$ Major sequelae (single) & $20.2 \%$ & - & - & Edmond et al. 2010 (27) \\
\hline$\%$ Major sequelae (multiple) & $4.5 \%$ & - & - & Edmond et al. 2010 (27) \\
\hline \multicolumn{5}{|l|}{ Disability weight for DALY calculations } \\
\hline All-cause acute otitis media & 0.02 & - & - & WHO GBD 1990 \\
\hline Pneumococcal pneumonia cases & 0.28 & - & - & WHO GBD 1990 \\
\hline Pneumococcal meningitis & 0.62 & - & - & WHO GBD 1990 \\
\hline Pneumococcal NPNM & 0.28 & - & - & Assumption (Griffiths $\mathrm{PhD}$ ) \\
\hline$\%$ Major sequelae (single) & 0.24 & - & - & Assumption (Griffiths PhD) \\
\hline$\%$ Major sequelae (multiple) & 0.63 & - & - & Assumption (Griffiths PhD) \\
\hline \multicolumn{5}{|l|}{ Mean duration of illness (in days) } \\
\hline All-cause acute otitis media & 6 & - & - & Assumption \\
\hline Pneumococcal pneumonia cases & 6 & - & - & Assumption \\
\hline Pneumococcal meningitis & 10 & - & - & Assumption \\
\hline Pneumococcal NPNM & 6 & - & - & Assumption \\
\hline \multicolumn{5}{|c|}{ Age distribution of disease cases and deaths } \\
\hline$<3 \mathrm{~m}:$ & $11.5 \%$ & - & - & Sanderson 2013, WHO IVR schedule report \\
\hline 3-5m: & $16.2 \%$ & - & - & Sanderson 2013, WHO IVR schedule report \\
\hline 6-8m: & $15.2 \%$ & - & - & Sanderson 2013, WHO IVR schedule report \\
\hline 9-11m: & $13.0 \%$ & - & - & Sanderson 2013, WHO IVR schedule report \\
\hline 12-23m: & $30.2 \%$ & - & - & Sanderson 2013, WHO IVR schedule report \\
\hline 24-35m: & $10.1 \%$ & - & - & Sanderson 2013, WHO IVR schedule report \\
\hline 36-47m: & $3.0 \%$ & - & - & Sanderson 2013, WHO IVR schedule report \\
\hline 48-59m: & $0.9 \%$ & - & - & Sanderson 2013, WHO IVR schedule report \\
\hline
\end{tabular}

${ }^{a}$ In the absence of vaccination, CFRs are assumed to decline in each successive birth cohort in line with the general trend in mortality among children under 5 . This is done by assuming that the fraction of deaths under age 5 caused by the disease remains fixed over time. 
Table 2. Input parameters for estimating PCV vaccine coverage and timeliness

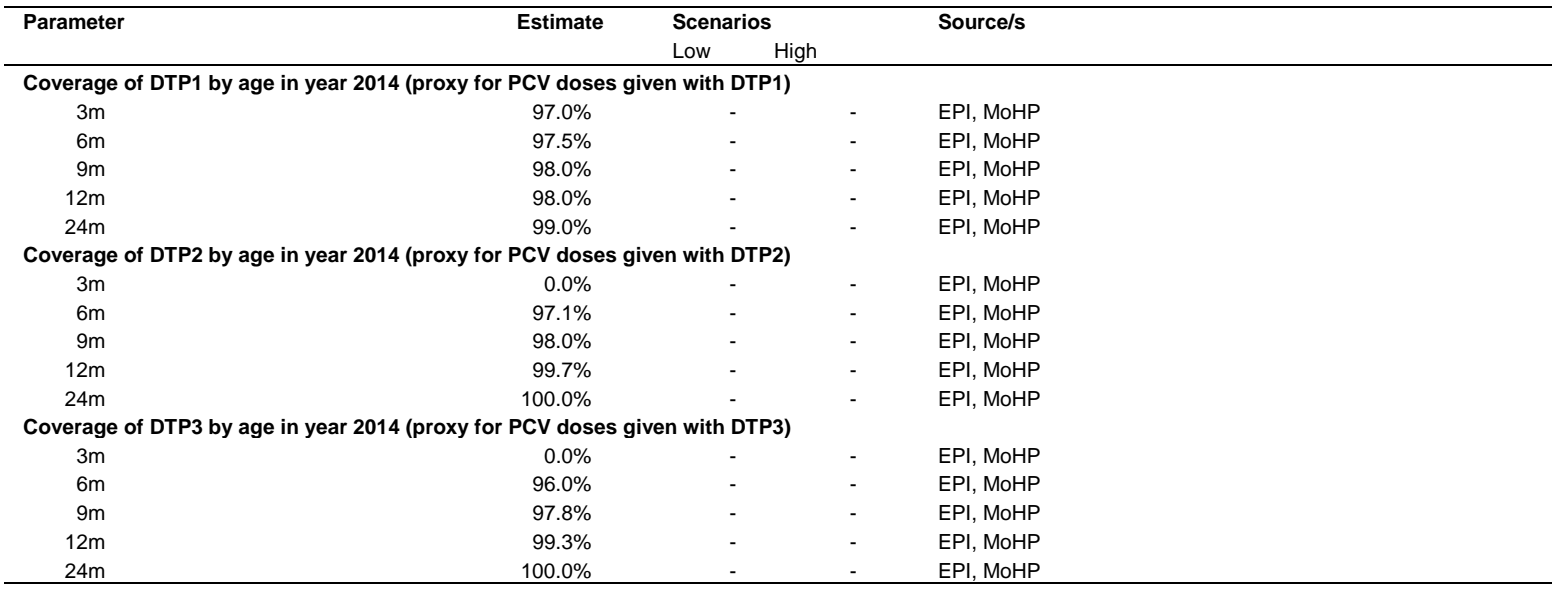

EPI: Expanded Program on Immunization; MoHP: Ministry of Health and Population

Coverage projections over the period 2014-2023 were estimated by assuming that PCV will achieve the same coverage and timeliness as DTP. 
Table 3. Input parameters for estimating the health impact of PCV13

\begin{tabular}{|c|c|c|c|c|}
\hline \multirow[t]{2}{*}{ Parameter } & \multirow[t]{2}{*}{ Estimate } & \multicolumn{2}{|c|}{ Scenarios } & \multirow[t]{2}{*}{ Source/s } \\
\hline & & Low & High & \\
\hline \multicolumn{5}{|c|}{ Vaccine efficacy vs all-cause acute otitis media } \\
\hline Dose 1 & $3.0 \%$ & $2.0 \%$ & $6.9 \%$ & Mahon et al. 2006 (33) \\
\hline Dose 2 & $5.5 \%$ & $3.7 \%$ & $9.0 \%$ & Mahon et al. 2006 (33) \\
\hline Dose 3 & $6.0 \%$ & $4.0 \%$ & $9.0 \%$ & Pavia et al. 2009 (31) \\
\hline \multicolumn{5}{|c|}{ Vaccine efficacy vs vaccine type pneumococcal pneumonia / meningitis / NPNM } \\
\hline Dose 1 & $41.0 \%$ & $31.9 \%$ & $69.2 \%$ & Mahon et al. 2006 (33) \\
\hline Dose 2 & $74.5 \%$ & $58.0 \%$ & $90.0 \%$ & Mahon et al. 2006 (33) \\
\hline Dose 3 & $81.0 \%$ & $63.0 \%$ & $90.0 \%$ & Lucero et al. 2009 (32) \\
\hline \multicolumn{5}{|l|}{$\%$ vaccine serotype coverage } \\
\hline Pneumococcal pneumonia cases & $74.0 \%$ & $67.0 \%$ & $79.0 \%$ & Johnson et al. 2010 (34) \\
\hline Pneumococcal meningitis & $74.0 \%$ & $67.0 \%$ & $79.0 \%$ & Johnson et al. 2010 (34) \\
\hline Pneumococcal NPNM & $74.0 \%$ & $67.0 \%$ & $79.0 \%$ & Johnson et al. 2010 (34) \\
\hline \multicolumn{5}{|l|}{ Other vaccination impact assumptions } \\
\hline$\%$ relative coverage ${ }^{\text {a }}$ & $99 \%$ & $80 \%$ & $99.7 \%$ & Assumption based on Egyptian DHS \\
\hline$\%$ decrease in dose efficacy per $\mathrm{yr}^{\mathrm{b}}$ & $5.0 \%$ & $0.0 \%$ & $10.0 \%$ & Assumption \\
\hline$\%$ contribution of herd effect in $<5 \mathrm{yrs}^{\circ}$ & $110 \%$ & $100 \%$ & $125 \%$ & Conservative assumption \\
\hline Decline in vaccine type coverage $/ \mathrm{yr}^{\mathrm{d}}$ & $5.5 \%$ & $0.0 \%$ & $6.0 \%$ & Assumption \\
\hline
\end{tabular}

DHS: Demographic and Health Survey

${ }^{a}$ Relative coverage is the coverage in those at risk of getting the disease (i.e. effective coverage) relative to coverage in the entire birth cohort (i.e., overall coverage). Overall coverage is multiplied by relative coverage to obtain a more realistic estimate of effective coverage.

${ }^{\mathrm{b}}$ To account for waning duration of clinical vaccine-induced protection, TRIVAC uses a waning matrix with age bands $(<3 \mathrm{~m}, 4-5 \mathrm{~m}, 6-8 \mathrm{~m}, 9-11 \mathrm{~m}, 12-23 \mathrm{~m}, 24-35 \mathrm{~m}, 36-$ $47 \mathrm{~m}, 48-59 \mathrm{~m}$ ) repeated in the rows and columns of the matrix. The direct protection at the start of each age band is represented by the diagonal from top-left to bottom-right of the matrix. Protection is re-calculated for each age band as the child gets older (moves from left to right in each row). Adjusted protection by age is calculated by adding together the revised protection estimates for each column.

${ }^{c}$ Rather than endogenous modeling of transmission dynamics, the \% of direct protection under 5 yrs is multiplied by a herd effect factor (e.g., $120 \%$ ) to give the $\%$ of total protection in the cohort of interest before age $5 \mathrm{yrs}$. This excludes any herd effect in individuals aged 5 yrs+ and is therefore very conservative.

${ }^{d}$ Vaccine type disease replacement is handled by reducing the expected vaccine type coverage in successive vaccinated cohorts by a fixed \% each year, thus reducing overall expected impact of the programme in each successive vaccinated cohort by a similar amount. Thus, for a given vaccinated cohort, the \% vaccine type coverage is equal to: $\left[T^{*}(1-R)^{\wedge} \mathrm{N}\right]$ where, $T=\%$ of disease caused by vaccine types in the year of vaccine introduction, $R=\%$ reduction in vaccine type coverage per year following vaccine introduction, $\mathrm{N}=$ number in the sequence of vaccinated birth cohorts. 
Table 4. Input parameters for estimating PCV13 program costs

\begin{tabular}{|c|c|c|c|c|}
\hline \multirow[t]{2}{*}{ Parameter } & \multirow[t]{2}{*}{ Estimate } & \multicolumn{2}{|c|}{ Scenarios } & \multirow{2}{*}{ Source/s } \\
\hline & & Low & High & \\
\hline \multicolumn{5}{|l|}{ Vaccine dose price projection } \\
\hline 2014 & $\$ 14.24$ & $\$ 10.00$ & $\$ 20.00$ & Local manufacturing company (Vacsera) \\
\hline 2015 & $\$ 13.81$ & $\$ 9.70$ & $\$ 19.40$ & Model derived \\
\hline 2016 & $\$ 13.40$ & $\$ 9.41$ & $\$ 18.82$ & Model derived \\
\hline 2017 & $\$ 13.00$ & $\$ 9.13$ & $\$ 18.25$ & Model derived \\
\hline 2018 & $\$ 12.61$ & $\$ 8.85$ & $\$ 17.71$ & Model derived \\
\hline 2019 & $\$ 12.23$ & $\$ 8.59$ & $\$ 17.17$ & Model derived \\
\hline 2020 & $\$ 11.86$ & $\$ 8.33$ & $\$ 16.66$ & Model derived \\
\hline 2021 & $\$ 11.51$ & $\$ 8.08$ & $\$ 16.16$ & Model derived \\
\hline 2022 & $\$ 11.16$ & $\$ 7.84$ & $\$ 15.67$ & Model derived \\
\hline 2023 & $\$ 10.83$ & $\$ 7.60$ & $\$ 15.20$ & Model derived \\
\hline \multicolumn{5}{|l|}{ Other vaccine dose costs } \\
\hline International handling (\% of vaccine price) & $3.00 \%$ & - & - & Assumption \\
\hline International delivery (\% of vaccine price) & $1.00 \%$ & - & - & EPI assumption \\
\hline Wastage (\% of doses discarded etc) ${ }^{\mathrm{a}}$ & $1.00 \%$ & - & - & EPI assumption \\
\hline \multicolumn{5}{|l|}{ Safety box cost ( 80 syringes per box) } \\
\hline Price of each safety box & $\$ 0.60$ & - & - & CDCD, MoHP \\
\hline International handling (\%) & $3.00 \%$ & - & - & Assumption \\
\hline International delivery (\%) & $2.00 \%$ & - & - & Assumption \\
\hline Wastage (\% of doses discarded etc) ${ }^{\mathrm{a}}$ & $5.00 \%$ & - & - & Assumption \\
\hline \multicolumn{5}{|l|}{ Administering syringe cost per dose } \\
\hline Price of each syringe & $\$ 0.06$ & - & - & CDCD, MoHP \\
\hline International handling (\%) & $3.00 \%$ & - & - & Assumption \\
\hline International delivery $(\%)$ & $2.00 \%$ & - & - & Assumption \\
\hline Wastage (\% of doses discarded etc) ${ }^{a}$ & $10.0 \%$ & - & - & CDCD, MoHP \\
\hline \multicolumn{5}{|l|}{ Incremental system costs of introduction } \\
\hline Incremental system cost per dose & $\$ 1.00$ & - & - & EPI assumption \\
\hline
\end{tabular}

EPI: Expanded Program on Immunization; CDCD, MoHP: Communicable Disease Control Department, Ministry of Health and Population.

${ }^{\text {a }}$ The $\%$ wastage is converted into a factor [ $1 /(1-\%$ wastage $\left.)\right]$ which is multiplied by the expected number of doses required to meet the anticipated level of coverage. 
Table 5. Input parameters for estimating health service utilization and costs (all costs are presented in 2013 US \$)

\begin{tabular}{|c|c|c|c|c|}
\hline \multirow[t]{2}{*}{ Parameter } & \multirow[t]{2}{*}{ Estimate } & \multicolumn{2}{|c|}{ Scenarios } & \multirow[t]{2}{*}{ Source/s } \\
\hline & & Low & High & \\
\hline \multicolumn{5}{|l|}{ OUTPATIENT VISITS } \\
\hline \multicolumn{5}{|l|}{ Outpatient visits per disease episode } \\
\hline All-cause acute otitis media (AOM) & 0.50 & 0.48 & 0.53 & National experts consensus \\
\hline Pneumococcal pneumonia cases & 0.80 & 0.76 & 0.84 & National experts consensus \\
\hline Pneumococcal meningitis & 0.80 & 0.76 & 0.84 & National experts consensus \\
\hline Pneumococcal NPNM & 0.50 & 0.48 & 0.53 & National experts consensus \\
\hline \multicolumn{5}{|l|}{ Government cost per outpatient visit ${ }^{a}$} \\
\hline All-cause acute otitis media & $\$ 4.08$ & $\$ 3.67$ & $\$ 4.49$ & WHO-CHOICE \\
\hline Pneumococcal pneumonia cases & $\$ 3.91$ & $\$ 3.86$ & $\$ 4.72$ & Field survey \\
\hline Pneumococcal meningitis & $\$ 4.81$ & $\$ 3.86$ & $\$ 4.72$ & Field survey \\
\hline Pneumococcal NPNM & $\$ 4.36$ & $\$ 3.92$ & $\$ 4.79$ & WHO-CHOICE \\
\hline \multicolumn{5}{|l|}{ INPATIENT ADMISSIONS } \\
\hline \multicolumn{5}{|c|}{ Inpatient admissions per disease episode } \\
\hline Pneumococcal pneumonia cases & 0.24 & 0.23 & 0.25 & National experts consensus \\
\hline Pneumococcal meningitis & 1.00 & 0.95 & 1.05 & National experts consensus \\
\hline Pneumococcal NPNM & 0.75 & 0.71 & 0.79 & National experts consensus \\
\hline \multicolumn{5}{|c|}{ Government cost per inpatient admission ${ }^{\mathrm{b}}$} \\
\hline Pneumococcal pneumonia cases & $\$ 147$ & $\$ 138$ & $\$ 152$ & Field survey \\
\hline Pneumococcal meningitis & $\$ 270$ & $\$ 238$ & $\$ 263$ & Field survey \\
\hline Pneumococcal NPNM & $\$ 145$ & $\$ 138$ & $\$ 152$ & Field survey \\
\hline
\end{tabular}

WHO-CHOICE: WHO initiative aimed at providing policy makers with evidence for deciding on interventions and programs that maximize health with available resources. (Available at http://www.who.int/choice/en/)

${ }^{a}$ Government costs per outpatient visit include the cost of visit, treatment, laboratory tests, and X-rays. Outpatient visits are distributed as follows: $25 \%$ public clinic, $25 \%$ primary hospital, $25 \%$ secondary hospital, $25 \%$ tertiary hospital for $\mathrm{AOM} ; 10 \% / 30 \% / 30 \% / 30 \%$ for pneumonia and meningitis; and $5 \% / 30 \% / 30 \% / 35 \%$ for NPNM. The cost presented is the weighted average of the provider-specific costs.

${ }^{\mathrm{b}}$ Government costs per inpatient admission include the cost per bed-day multiplied by the expected length of stay and the cost of any disease-specific drugs and diagnostics. Inpatient admissions are distributed as follows: $20 \%$ primary hospital, $40 \%$ secondary hospital, $40 \%$ tertiary hospital for pneumonia; $10 \% / 40 \% / 50 \%$ for meningitis; and $33.3 \% / 33.3 \% / 33.3 \%$ for NPNM. The cost presented is the weighted average of the provider-specific costs. 
Table 6. Discounted health benefits (10 cohorts vaccinated over the period 2014-2023)

\begin{tabular}{|c|c|c|c|}
\hline & $\begin{array}{l}\text { No vaccine } \\
\text { (status quo) }\end{array}$ & $\begin{array}{l}\text { PCV13 } \\
\text { With vaccine }\end{array}$ & Averted \\
\hline Total cases $<5$ yrs & $37,748,986$ & $35,892,051$ & $1,856,935$ \\
\hline All-cause acute otitis media & $36,958,144$ & $35,428,252$ & $1,529,891$ \\
\hline Pneumococcal pneumonia cases & 667,159 & 391,263 & 275,896 \\
\hline Pneumococcal meningitis & 17,656 & 10,355 & 7,302 \\
\hline Pneumococcal NPNM & 106,027 & 62,181 & 43,846 \\
\hline Total outpatient visits & $19,079,938$ & $18,066,511$ & $1,013,427$ \\
\hline All-cause acute otitis media & $18,479,072$ & $17,714,126$ & 764,946 \\
\hline Pneumococcal pneumonia cases & 533,727 & 313,011 & 220,717 \\
\hline Pneumococcal meningitis & 14,125 & 8,284 & 5,841 \\
\hline Pneumococcal NPNM & 53,013 & 31,090 & 21,923 \\
\hline Total inpatient admissions & 257,295 & 150,893 & 106,401 \\
\hline Pneumococcal pneumonia cases & 160,118 & 93,903 & 66,215 \\
\hline Pneumococcal meningitis & 17,656 & 10,355 & 7,302 \\
\hline Pneumococcal NPNM & 79,520 & 46,635 & 32,885 \\
\hline Total deaths $<5$ yrs & 20,505 & 11,922 & 8,583 \\
\hline Pneumococcal pneumonia cases & 14,878 & 8,651 & 6,228 \\
\hline Pneumococcal meningitis & 1,244 & 723 & 521 \\
\hline Pneumococcal NPNM & 4,383 & 2,548 & 1,834 \\
\hline Total children with permanent disability & 4,054 & 2,376 & 1,678 \\
\hline Sequelae group $\mathrm{A}$ & 3,315 & 1,943 & 1,372 \\
\hline Sequelae group $B$ & 739 & 433 & 306 \\
\hline DALYs Lost & 665,348 & 392,470 & 272,878 \\
\hline YLDs - DALYs due to morbidity & 57,255 & 38,766 & 18,488 \\
\hline YLLs - DALYs due to mortality & 608,094 & 353,704 & 254,390 \\
\hline
\end{tabular}

Health benefits are discounted at $3 \%$ per year. 
Table 7. Discounted economic benefits (10 cohorts vaccinated over the period 2014-2023)

\begin{tabular}{|c|c|c|c|}
\hline & $\begin{array}{l}\text { No vaccine } \\
\text { (status quo) }\end{array}$ & $\begin{array}{l}\text { PCV13 } \\
\text { With vaccine }\end{array}$ & Averted \\
\hline TOTAL GOV. HEALTH SERVICE COSTS ${ }^{a}$ & $\$ 117,656,726$ & $\$ 97,074,939$ & $\$ 20,581,788$ \\
\hline Total outpatient visit costs & $\$ 77,821,729$ & $\$ 73,713,247$ & $\$ 4,108,482$ \\
\hline All-cause acute otitis media & $\$ 75,438,151$ & $\$ 72,315,370$ & $\$ 3,122,781$ \\
\hline Pneumococcal pneumonia cases & $\$ 2,084,637$ & $\$ 1,222,559$ & $\$ 862,078$ \\
\hline Pneumococcal meningitis & $\$ 67,883$ & $\$ 39,811$ & $\$ 28,072$ \\
\hline Pneumococcal NPNM & $\$ 231,059$ & $\$ 135,507$ & $\$ 95,552$ \\
\hline Total inpatient admission costs & $\$ 39,834,997$ & $\$ 23,361,692$ & $\$ 16,473,305$ \\
\hline Pneumococcal pneumonia cases & $\$ 23,537,378$ & $\$ 13,803,766$ & $\$ 9,733,612$ \\
\hline Pneumococcal meningitis & $\$ 4,767,225$ & $\$ 2,795,794$ & $\$ 1,971,431$ \\
\hline Pneumococcal NPNM & $\$ 11,530,394$ & $\$ 6,762,132$ & $\$ 4,768,262$ \\
\hline
\end{tabular}

Costs are discounted at $3 \%$ per year.

${ }^{a}$ Government perspective includes all bed-days and disease-specific drug/diagnostic costs borne by the government at the following health providers: public clinic, public primary, secondary and tertiary hospitals. 
Table 8. Discounted cost-effectiveness of PCV (10 cohorts vaccinated over the period 2014-2023)

\begin{tabular}{cr}
\hline & $\begin{array}{l}\text { PCV13 } \\
\text { Government } \\
\text { perspective }\end{array}$ \\
\hline Cost-effectiveness compared to no vaccine & $\$ 1,068,599,533$ \\
Net cost of vaccine introduction & $\$ 1,089,181,321$ \\
Costs of vaccine introduction & $\$ 20,581,788$ \\
Health service costs avoided & $\mathbf{2 7 2 , 8 7 8}$ \\
DALYs averted & 18,488 \\
YLDs averted - DALYS due to morbidity & 254,390 \\
YLLs averted - DALYs due to mortality & $\$ 3,916$ \\
US\$ per DALY averted & \\
Cost-effectiveness threshold & $\$ 3,187$ \\
1 x GDP per capita (2012) - WHO threshold for 'highly cost-effective' & $\$ 9,562$ \\
$3 \times$ GDP per capita (2012) - WHO threshold for 'cost-effective' & \\
\hline
\end{tabular}

Costs and DALYs are discounted at $3 \%$ per year. 
Figure 1. Cost-effectiveness of introducing PCV13 and sensitivity analysis (government perspective)

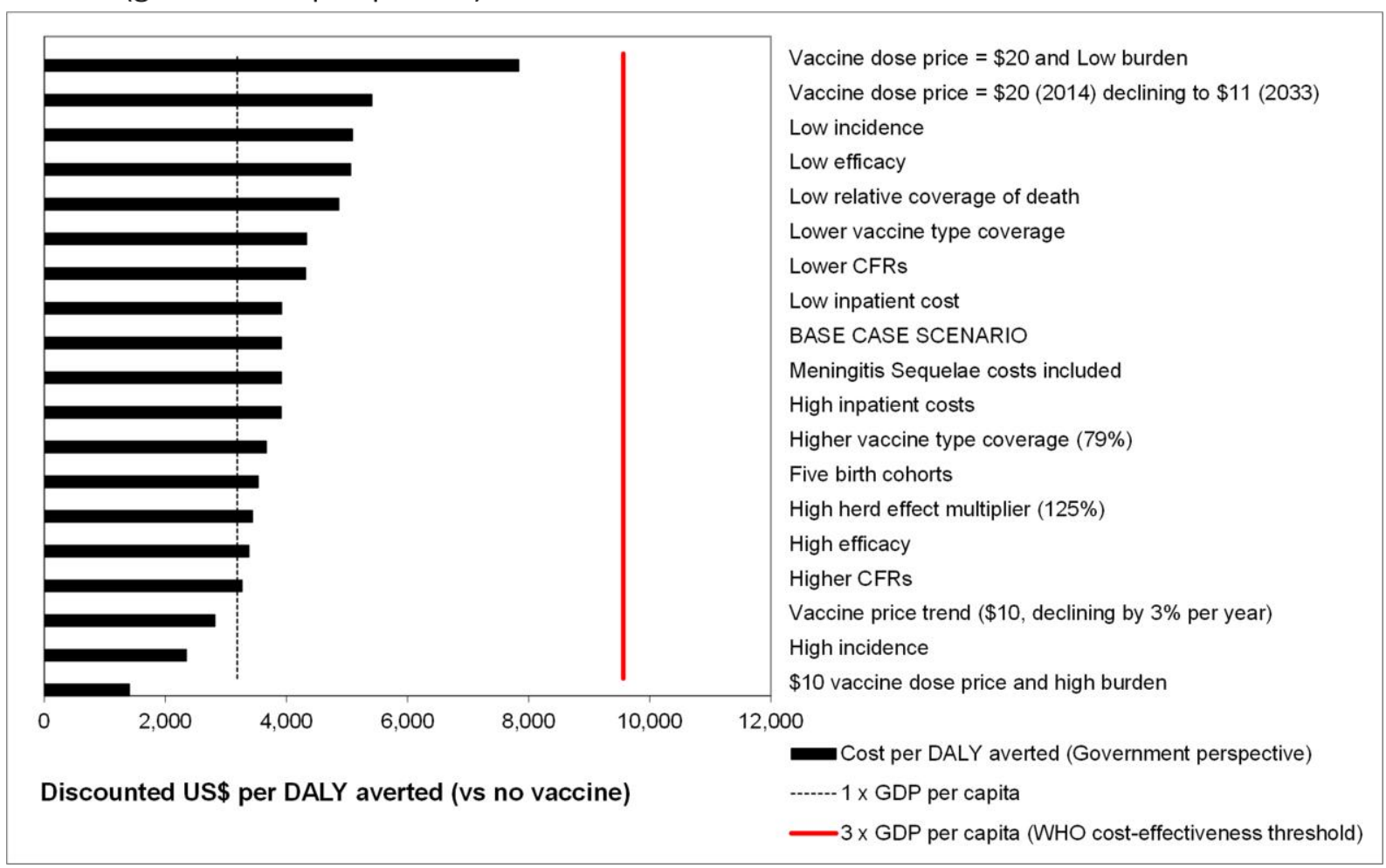




\section{ABSTRACT}

Introduction: Pneumonia is one of the most important causes of morbidity and mortality in children under 5 in Egypt, and the Ministry of Health of Egypt is considering introducing pneumococcal conjugate vaccine $(\mathrm{PCV})$ in its national immunization program. We performed an economic analysis to evaluate the cost-effectiveness of this vaccine in Egypt and to provide the decision-makers with needed evidence.

Methods: The analysis was done using the TRIVAC model. Data included demographic characteristics, burden of disease, coverage and efficacy of the vaccine, health resource utilization, and costs of pneumococcal disease vaccination and treatment. Whenever possible, we used national or regional data. Two alternatives were compared: (1) general vaccination of children younger than 5 years with the 13 -valent pneumococcal conjugate vaccine (PCV13), using a three-dose schedule without booster, and (2) no vaccination. Outcomes of 10 cohorts from birth to 5 years were analyzed. The study was performed from the governmental perspective and selected public health providers.

Results: In comparison to no vaccine, the introduction of PCV13 would be cost-effective, with an incremental cost-effectiveness ratio of US\$3,916 per disability-adjusted life-year (DALY) averted (government perspective). The total incremental cost of the PCV vaccination program (10 cohorts) would be approximately US\$ 1.09 billion. Over the 10 cohorts, the program would avert 8,583 pneumococcal deaths $-42 \%$ of all pneumococcal-related deaths. Conclusion: The introduction of PCV13 would be a good value for money from the government perspective. It would represent a high-impact public health intervention for Egypt and respond to the National Immunization Technical Advisory Group (NITAG) resolution on reducing pneumonia burden and overall child mortality. Strengthening surveillance will be critical to generating high-quality national data, improving future economic analyses that 
support evidence-based decisions for introducing vaccines and public health interventions, and to monitoring their impact.

Keywords: pneumococcal conjugate vaccine, cost-effectiveness, Egypt, immunization program, vaccine 